\title{
Consecutive Integers with Equally Many Principal Divisors
}

\author{
Roger B. Eggleton, \\ Mathematics Department, \\ Illinois State University, \\ Normal, IL 61790, U.S.A. \\ James A. MacDougall, \\ School of Mathematical and Physical Sciences, \\ The University of Newcastle, \\ Newcastle, N.S.W. 2308, Australia.
}

May 28, 2008

\section{Introduction}

Classifying the positive integers as primes, composites, and the unit, is so familiar that it seems inevitable. However, other classifications can bring interesting relationships to our attention. In that spirit, let us classify positive integers by the number of principal divisors they possess, where we define a principal divisor of a positive integer $n$ to be any prime-power divisor $p^{a} \mid n$ which is maximal (so $p$ is prime, $a$ is a positive integer, and $p^{a+1}$ is not a divisor of $n$ ). The standard notation $p^{a}|| n$ can be read as " $p^{a}$ is a principal divisor of $n$ ".

The Fundamental Theorem of Arithmetic is usually stated in a form emphasizing how primes enter the structure of the positive integers, such as: Every positive integer is the product of a unique finite multiset of primes. (Recall that a multiset is a collection of elements in which multiple occurrences are permitted.) Alternatively, the Fundamental Theorem of Arithmetic can be stated in a form that focuses on how maximal prime-powers enter the structure of the positive integers, such as: Every positive integer is the product of a unique finite set of powers of distinct primes. Consequently every positive integer is the product of its principal divisors, and every finite set of powers of distinct primes is the set of principal divisors of a unique 
positive integer. Of course, the number of principal divisors of $n$ is equal to the number of distinct prime factors of $n$, but here the principal divisors are the simple structural components of $n$, whereas the distinct prime factors are but a shadow of that structure. Readers who find the present paper of interest might find similar interest in [6], where upper bounds on the sum of principal divisors of $n$ are established by elementary means.

For each integer $n \geq 0$, let $P_{n}$ be the set of all positive integers with exactly $n$ principal divisors, so $P_{0}=\{1\}$, and

$$
\begin{aligned}
& P_{1}=\{2,3,4,5,7,8,9,11,13,16,17,19,23,25,27,29,31,32,37, \cdots\}, \\
& P_{2}=\{6,10,12,14,15,18,20,21,22,24,26,28,33,34,35,36,38, \cdots\}, \\
& P_{3}=\{30,42,60,66,70,78,84,90,102,105,110, \cdots\}, \\
& P_{4}=\{210,330,390,420,462,510, \cdots\}, \text { etc. }
\end{aligned}
$$

In particular, $P_{1}$ comprises the prime-powers, or principal integers; $P_{2}$ comprises the products of two coprime principal integers, or rank 2 integers; and so on. Collectively, we call $\left\{P_{n}: n \geq 0\right\}$ the rank sets of positive integers.

Clearly the rank sets are a partition of the positive integers, by the Fundamental Theorem of Arithmetic. Thus it is interesting to look at the occurrence of runs of consecutive integers within each rank set: this is one of the relationships immediately brought into focus by the classification.

For compactness, let us write $a^{[r]}$ to denote the run of $r$ consecutive integers beginning with $a$, where $a$ and $r$ are positive integers, so

$$
a^{[r]}=\{a+i: 0 \leq i<r\} .
$$

We call $r$ the size of the run. In particular, the run $a^{[r]}$ is nontrivial if $r \geq 2$, and $a^{[r]} \subset P_{n}$ is a maximal run in $P_{n}$ if it is nontrivial and $P_{n}$ contains neither $a-1$ nor $a+r$. Thus, the first few maximal runs in $P_{1}$ are $2^{[4]}, 7^{[3]}, 16^{[2]}, 31^{[2]}, 127^{[2]}$ and $256^{[2]}$; the first few maximal runs in $P_{2}$ are $14^{[2]}, 20^{[3]}, 33^{[4]}, 38^{[3]}, 44^{[3]}, 50^{[3]}$ and $54^{[5]}$.

It is easy to see that the size of runs in $P_{n}$ is bounded. For if $M$ is the product of the first $n+1$ primes, then $M \in P_{n+1}$ and any run of $M$ consecutive integers contains a multiple of $M$, so any run in $P_{n}$ has size less than $M$. Thus, for each integer $n \geq 0$ there is a positive integer $r(n)$ which is the maximum size attained by runs in $P_{n}$. Trivially, $r(0)=1$. We have already seen that $r(1) \geq 4$ and $r(2) \geq 5$, and we shall soon see that in fact $r(1)=4$. Our main objective is to study $r(2)$, which we shall determine "within 1". Later we shall also discuss $r(n)$ for $n \geq 3$. 


\section{Maximal runs in $P_{1}$ and $P_{2}$}

Returning to the principal integers, it is clear that any nontrivial run in $P_{1}$ contains an even integer so, being principal, any such integer must be a power of 2 . Since 2 and 4 are the only powers of 2 that differ by 2 , any maximal run of principal integers greater than 5 must contain exactly one even integer, so $2^{[4]}$ is the unique longest run in $P_{1}$, and $r(1)=4$.

The long-standing conjecture credited to Catalan, that 8 and 9 are the only two consecutive integers which are nontrivial prime powers, was recently proved by Mihailescu [11]. From this it follows that any maximal run of principal integers greater than 9 must contain a power of 2 and any adjacent number in the run must be a prime. It is well known that $2^{n}-1$ can only be prime when $n$ itself is prime, and $2^{n}+1$ can only be prime when $n$ is a power of 2 : when $n \geq 3$ these two conditions are mutually exclusive, so any maximal run of principal integers greater than 9 has just two members.

Primes of the form $2^{n}+1$ are Fermat primes. The only known Fermat primes are $3,5,17,257$ and 65537 , but no proof is known that there are no others. Primes of the form $2^{n}-1$ are Mersenne primes. Currently 44 Mersenne primes are known [7]. A distributed computing project known as GIMPS (the Great International Mersenne Prime Search) has made numerous additions to this list in recent years, but no proof is known that infinitely many such primes exist. Consequently, although we know $2^{[4]}$ and $7^{[3]}$ are the only maximal runs of more than two principal integers, it is not known whether $P_{1}$ contains infinitely many runs of size 2 .

What happens with maximal runs of rank 2 numbers? This is less familiar territory, so one does not know quite what to expect. We shall prove:

Theorem 1 There is no run of 10 consecutive integers in $P_{2}$.

Hence $r(2) \leq 9$. Is this a "sharp" result? It turns out to be "within 1 " of the exact value of $r(2)$. Our methods appear unable to decide on the existence of runs of size 9 in $P_{2}$, but our results strongly suggest the following:

Conjecture 1 In $P_{2}$ there is no run of size 9 and the only maximal runs of size greater than 6 are $141^{[8]}, 212^{[8]}, 323^{[7]}$ and $2302^{[7]}$.

It may also be true that $91^{[6]}$ is the only maximal run of size 6 in $P_{2}$, but we have less information about runs of size 6 than about longer runs. 


\section{Størmer's Theorems}

For any set of primes $P$ let $S(P)$ be the set of positive integers with all their prime factors in $P$, that is, $S(P)$ is the multiplicative semigroup of positive integers generated by $P$. Some results about the integers in $S(P)$ turn out to be among the main tools we require to prove Theorem 1 .

If $D$ is a non-square integer, the Pell equation $x^{2}-D y^{2}=c$ always has positive integer solutions when $c=1$ (see [12], for example). However, for other values of $c$ it need not have positive integer solutions; for instance, this is the case when $c=-1$ if $D \equiv 3(\bmod 4)$. But if $c \in\{1,-1\}$ and $x^{2}-D y^{2}=c$ does have positive integer solutions, then all such solutions are generated by the smallest positive solution $\left(x_{0}, y_{0}\right)$, usually called the fundamental solution or minimal solution $[12,16]$. In 1897 Carl Størmer published the following theorem [13] which identifies a remarkable property of the fundamental solution.

Størmer's Pell Equation Theorem Let $D$ be a non-square positive integer, let $P$ be the set of prime divisors of $D$, and let $c \in\{1,-1\}$. If the Pell equation $x^{2}-D y^{2}=c$ has positive integer solutions and $\left(x_{0}, y_{0}\right)$ is the fundamental solution, then $y_{0}$ is the only solution for $y$ that is a possible member of $S(P)$.

For example, if $D=2$ then $S(P)$ comprises all the powers of 2 . Thus the fundamental solution $(x, y)=(3,2)$ is the only solution to $x^{2}-2 y^{2}=1$ in positive integers with $y$ a power of 2 ; similarly $(1,1)$ is the only solution to $x^{2}-2 y^{2}=-1$ in positive integers with $y$ a power of 2 .

Størmer used his Theorem to prove the following result [14], first published in 1898.

Størmer's Neighboring Pairs Theorem For given positive integers $A, B$, $m, n, a_{1}, \cdots, a_{m}, b_{1}, \cdots, b_{n}$, there are at most finitely many sequences of positive integers $x_{1}, \cdots, x_{m}, y_{1}, \cdots, y_{n}$ such that

$$
\left|A a_{1}^{x_{1}} \cdots a_{m}^{x_{m}}-B b_{1}^{y_{1}} \cdots b_{n}^{y_{n}}\right| \leq 2 .
$$

All solutions follow from the fundamental solutions to a finite set of Pell equations determined by $A, B, m, n, a_{1}, \cdots, a_{m}, b_{1}, \cdots, b_{n}$.

In principle it is routine to set up the Pell equations referred to in the Neighboring Pairs Theorem, and to determine the fundamental solutions of those that do have solutions, so Størmer effectively provided an algorithm 
for solving the Diophantine inequality in the Neighboring Pairs Theorem. Subsequently he gave simpler proofs of both theorems in a paper [15] that we commend to the reader. We shall soon solve two special cases of the neighboring pairs problem by methods illustrating Størmer's arguments: the results are stated below as a Corollary to the Neighboring Pairs Theorem.

If $P$ is finite, then by Størmer's Neighboring Pairs Theorem there are only finitely many pairs of consecutive integers in $S(P)$. D. H. Lehmer [9] gave a new proof of Størmer's theorem for the case of consecutive integers, and explicitly computed the last pairs of consecutive integers in $S(P)$ when $P$ is any initial subset of the primes with largest member 41 or less [10]. Some related tabulations are given in [4], and some elementary arguments establishing special instances of Størmer's result are given in [5] and [8]. The latter paper, by Halsey and Hewitt, discusses the fascinating connection between fundamental frequency ratios in Western music and consecutive pairs of integers in $S(2,3,5)$.

\begin{tabular}{|l|l|}
\hline$S(P)$ & Last maximal runs \\
\hline$S(2,3)$ & $8^{[2]}, 2^{[3]}$ \\
$S(2,3,5)$ & $80^{[2]}, 8^{[3]}, 3^{[4]}$ \\
$S(2,3,5,7)$ & $4374^{2]}, 48^{3]}, 7^{[4]}$ \\
\hline
\end{tabular}

Table 1: Last maximal runs in $S(P)$

For our present purposes, we note in Table 1 the last maximal runs of various sizes in $S(P)$ when $P$ is an initial subset of the primes with largest member 7 or less. We shall also need the following consequence of Størmer's theorem:

Corollary 1 The only pairs of integers satisfying $\left|3^{a}-5^{b}\right|=2$ are $\{3,5\}$ and $\{25,27\}$, and the only pair satisfying $\left|3^{a}-7^{b}\right|=2$ is $\{7,9\}$.

Proof Størmer's method applied to pairs of integers $\left\{3^{a}, 5^{b}\right\}$ satisfying $\left|3^{a}-5^{b}\right|=2$ puts them equal to $x-1$ and $x+1$, so their product is of the form $x^{2}-1=D y^{2}$, where $y \in S(3,5)$ and $D \in\left\{3^{a} 5^{b} \mid 1 \leq a, b \leq 2\right\}$ is non-square, so $D \in\{15,45,75\}$. For these three values of $D$, the corresponding Pell equations have fundamental solutions $\left(x_{0}, y_{0}\right)=(4,1),(161,24)$ and $(26,3)$ respectively. Now Størmer's Pell Equation Theorem shows that $y_{0}=1$ and 3 are the only $y$ solutions of these Pell equations that lie in $S(3,5)$. The corresponding $x$ solutions yield the pairs $\left\{x_{0}-1, x_{0}+1\right\}=\{3,5\}$ and $\{25,27\}$, so these are the only pairs $\left\{3^{a}, 5^{b}\right\}$ that differ by 2 . 
Similarly, pairs of integers $\left\{3^{a}, 7^{b}\right\}$ satisfying $\left|3^{a}-7^{b}\right|=2$ must correspond to fundamental solutions of $x^{2}-1=D y^{2}$, where $y \in S(3,7)$ and $D$ is non-square, so $D \in\{21,63,147\}$. The corresponding Pell equations have fundamental solutions $\left(x_{0}, y_{0}\right)=(55,12),(8,1)$ and $(97,8)$ respectively. Now $y_{0}=1$ is the only $y$ solution of these Pell equations that lies in $S(3,7)$. The corresponding $x$ solution yields the pair $\{x-1, x+1\}=\{7,9\}$, so this is the only pair $\left\{3^{a}, 7^{b}\right\}$ differing by 2 .

\section{Constraints on runs in $P_{2}$}

This section builds a proof of Theorem 1. Our method is to establish several properties of runs in $P_{2}$ that allow us to close in on the possible structure of long runs. Finally we accumulate enough constraints to show that no run of size 10 or more could possibly satisfy all the constraints, so we can conclude that every run in $P_{2}$ has size less than 10. Consequences of Størmer's Neighboring Pairs Theorem are used in several key steps, including some of the results in Table 1 and our Corollary to the Neighboring Pairs Theorem.

Property 1 Any run of consecutive integers in $P_{2}$ contains at most one multiple of 6 .

Proof Since 89 and 97 are the first two consecutive primes with difference greater than 6 , it follows that between any two consecutive multiples of 6 less than 96 there is at least one prime. Since primes are in $P_{1}$, no run in $P_{2}$ can contain two consecutive multiples of 6 less than 96 . On the other hand, 8 and 9 are the last two consecutive integers in $S(2,3)$, so 48 and 54 are the last two consecutive multiples of 6 in $P_{2}$. The property follows.

The next three properties concern multiples of 5 in $P_{2}$ that occur within runs which contain a multiple of 6 .

Property 2 Only one maximal run of consecutive integers in $P_{2}$ contains a multiple of 6 and a multiple of 5 which differ by 2 or 3 ; that run is $158^{[5]}$.

Proof If $6 a$ and $5 b$ are members of $P_{2}$ that differ by 2 , then $6 a \in S(2,3)$ and $5 b=10 c \in S(2,5)$ for some integer $c$. Then $3 a$ and $5 c$ are consecutive integers in $S(2,3,5)$, and $80^{[2]}$ is the last such pair. Hence the corresponding pairs $\{6 a, 5 b\}$ in $P_{2}$ are $\{10,12\},\{18,20\},\{48,50\}$ and $\{160,162\}$. For the first three pairs, the intervening number is in $P_{1}$; hence the only maximal run in $P_{2}$ that contain integers $6 a$ and $5 b$ with $|6 a-5 b|=2$ is $158^{[5]}$. 
Similarly, if $6 a$ and $5 b$ are members of $P_{2}$ that differ by 3 , then $6 a \in$ $S(2,3)$ and $5 b=15 c \in S(3,5)$ for some integer $c$, so $2 a$ and $5 c$ are consecutive integers in $S(2,3,5)$. The corresponding pairs $\{6 a, 5 b\}$ in $P_{2}$ are $\{12,15\}$, $\{15,18\},\{45,48\}$ and $\{72,75\}$, but in each case there is an intervening prime, so no maximal run in $P_{2}$ contains integers $6 a$ and $5 b$ with $|6 a-5 b|=3$.

Property 3 No run of consecutive integers in $P_{2}$ contains a multiple of 6 and a multiple of 5 which differ by 4 .

Proof First suppose $6 a$ and $20 b$ are members of $P_{2}$ that differ by 4 , so $6 a=12 c \in S(2,3)$ for some integer $c$, and $20 b \in S(2,5)$. Then $5 b$ and $3 c$ are consecutive integers in $S(2,3,5)$. Since $80^{[2]}$ is the last such pair, the corresponding pairs $\{6 a, 20 b\}$ in $P_{2}$ are $\{20,24\},\{36,40\},\{96,100\}$ and $\{320,324\}$. The first three pairs have an intervening prime, while the last pair is not in a run in $P_{2}$ because $322 \in P_{3}$. Thus, no run of consecutive integers in $P_{2}$ contains a multiple of 6 and a multiple of 20 differing by 4 .

Now suppose $6 a$ and $10 d$ are members of $P_{2}$ that differ by 4 , and $d$ is odd. Then $|3 a-5 d|=2$, so $a$ is also odd. But $6 a \in S(2,3)$ and $10 d \in S(2,5)$, so $a$ is a power of 3 and $d$ is a power of 5 . By Corollary $1,\{3,5\}$ and $\{25,27\}$ are the only pairs of proper powers of 3 and 5 that differ by 2 . The corresponding pairs $\{6 a, 10 d\}$ in $P_{2}$ are $\{6,10\}$ and $\{50,54\}$, but each has an intervening prime, so neither pair is contained in a run in $P_{2}$. The property follows.

It is noteworthy that the pair $\{320,324\}$, appearing in the proof of Property 3 , actually corresponds to a near miss: $P_{2}$ contains the two maximal runs $319^{[3]}$ and $323^{[7]}$, and the only intervening integer is $322 \in P_{3}$. The two bordering integers are $318 \in P_{3}$ and $330 \in P_{4}$ (both multiples of 6 ), and their neighbors 317 and 331 are consecutive primes.

Property 4 Any run of consecutive integers in $P_{2}$ contains at most one multiple of 5 .

Proof On the contrary, suppose there is a run of consecutive integers in $P_{2}$ that contains two multiples of 5 . Let $R$ be that portion of the run which begins and ends with two consecutive multiples of 5 . Since $R$ has size 6 , we have $6 a \in R$ for some integer $a$. But $6 a \in P_{2}$ so it is distinct from the multiples of 5 . If $6 a$ differs by 2 from the nearer multiple of 5 , these two members of $R$ belong to the maximal run $158^{[5]}$, by Property 2. But this does not contain two multiples of 5 , so is disjoint from $R$. Hence $6 a$ must be adjacent to the nearer multiple of 5 . But then it must differ by 4 from the 
other multiple of 5 in $R$, and Property 3 shows that no run in $P_{2}$ contains two such numbers. Hence, by contradiction, $R$ does not exist.

Since any run of 10 consecutive integers contains two multiples of 5 , Property 4 immediately implies our target result:

Theorem 1 There is no run of 10 consecutive integers in $P_{2}$.

Corollary 2 Runs in $P_{2}$ have maximum size $r(2)=8$ or 9 .

Although our methods do not seem to be strong enough to decide whether runs of size 9 exist in $P_{2}$, consideration of multiples of 7 yields further properties, revealing more of the structure of $P_{2}$. In particular, we are led to discover the examples of runs of size 8 in $P_{2}$ which are incorporated in Conjecture 1. We shall pursue this in the next section.

\section{$5 \quad$ Further constraints on runs in $P_{2}$}

Now that we know $P_{2}$ has no runs of size 10 or more, the study of long runs of rank 2 integers can proceed by asking: What is the structure of any run of size 7 or more in $P_{2}$ ? Since any run of 7 consecutive integers must contain a multiple of 7 , at least one multiple of 6 , and at least one multiple of 5 , the relative placement of these multiples will now be considered.

Property 5 Only two maximal runs of consecutive integers in $P_{2}$ contain a multiple of 6 and a multiple of 7 which differ by 2 or 3 : those runs are $54^{[5]}$ and $141^{[8]}$.

Proof If $6 a$ and $7 b$ are members of $P_{2}$ that differ by 2 , then $6 a \in S(2,3)$ and $7 b=14 c \in S(2,7)$ for some integer $c$. Then $3 a$ and $7 c$ are consecutive integers in $S(2,3,7)$. From the fact that $4374^{[2]}$ is the last pair in $S(2,3,5,7)$, a straightforward calculation verifies that $63^{[2]}$ is the last pair in $S(2,3,7)$. The corresponding pairs $\{6 a, 7 b\}$ in $P_{2}$ are $\{12,14\},\{54,56\}$ and $\{96,98\}$. For the first and third pair, the intervening number is prime; hence the only maximal run in $P_{2}$ that contains integers $6 a$ and $7 b$ with $|6 a-7 b|=2$ is $54^{[5]}$.

Similarly, if $6 a$ and $7 b$ are members of $P_{2}$ that differ by 3 , then $6 a \in$ $S(2,3)$ and $7 b=21 c \in S(3,7)$ for some integer $c$, so $2 a$ and $7 c$ are consecutive integers in $S(2,3,7)$. The corresponding pairs $\{6 a, 7 b\}$ in $P_{2}$ are $\{18,21\}$, $\{21,24\},\{81,84\},\{144,147\}$ and $\{189,192\}$. For all but one of these pairs, there is an intervening prime; hence the only maximal run in $P_{2}$ that contains integers $6 a$ and $7 b$ with $|6 a-7 b|=3$ is $141^{[8]}$. 
Property 6 Any run of consecutive integers in $P_{2}$ contains at most one multiple of 7 .

Proof On the contrary, suppose there is a run $R$ of consecutive integers in $P_{2}$ that contains two consecutive multiples of 7 . Since they are in $P_{2}$, neither is a multiple of 6 , so there is a multiple of 6 between them but not adjacent to either of them. Thus the multiple of 6 and the nearer multiple of 7 differ by 2 or 3 , so $R$ must be contained in $54^{[5]}$ or $141^{[8]}$, by Property 5 . But each of these runs contains only one multiple of 7 , so by contradiction it follows that $R$ does not exist.

Property 7 There are no runs of consecutive integers in $P_{2}$ that contain a multiple of 6 and a multiple of 7 which differ by 4 .

Proof First suppose $6 a$ and $28 b$ differ by 4 and belong to some run of consecutive integers in $P_{2}$. Then $6 a=12 c \in S(2,3)$ for some integer $c$, and $28 b \in S(2,7)$, so $7 b$ and $3 c$ are consecutive integers in $S(2,3,7)$. As noted in the proof of Property $5,63^{[2]}$ is the last pair in $S(2,3,7)$. The corresponding pairs in $P_{2}$ are $\{24,28\},\{108,112\}$ and $\{192,196\}$, but none of them is contained in a run in $P_{2}$.

Now suppose, for some odd integer $d$, that $6 a \in S(2,3)$ and $14 d \in S(2,7)$ differ by 4 and belong to some run of consecutive integers in $P_{2}$. Then $|3 a-7 d|=2$, so $a$ is odd; hence $3 a$ is a power of 3 and $7 d$ is a power of 7. By our corollary to the Neighboring Pairs Theorem, $\{7,9\}$ is the only pair of proper powers of 3 and 7 that differ by 2 ; the corresponding pair $\{6 a, 14 d\}=\{14,18\}$ in $P_{2}$ is not contained in a run in $P_{2}$. The property follows.

Property 8 Exactly two maximal runs of consecutive integers in $P_{2}$ contain a multiple of 6 and a multiple of 35 : these are $33^{[4]}$ and $4374^{[2]}$.

Proof If $6 a$ and $35 b$ are members of some run of consecutive integers in $P_{2}$, then $6 a \in S(2,3)$ and $35 b \in S(5,7)$, so $|6 a-35 b|$ cannot be a multiple of $2,3,5$ or 7 . But every run in $P_{2}$ has size less that 10 , by our Theorem, so $|6 a-35 b|=1$, and $6 a$ and $35 b$ are consecutive integers in $S(2,3,5,7)$. Since $4374^{[2]}$ is the last nontrivial run in $S(2,3,5,7)$, the corresponding pairs in $P_{2}$ are $\{35,36\}$ and $\{4374,4375\}$. The corresponding maximal runs in $P_{2}$ are $33^{[4]}$ and $4374^{[2]}$.

Property 9 Any run of size at least 7 in $P_{2}$ contains exactly one multiple of 6 , exactly one multiple of 5 , and exactly one multiple of 7 , and these are 
three distinct members of the run. The multiple of 5 is always adjacent to the multiple of 6 . If the run has size 8 or more, the multiple of 7 is also adjacent to the multiple of 6 , except in the case of the maximal run $141^{[8]}$.

Proof Uniqueness of the multiples of 6,5 and 7 follows from Properties 1,4 and 6 respectively. By Property 8 , the only two runs in $P_{2}$ containing a multiple of 6 , and a multiple of 5 which is also a multiple of 7 , have size less than 7 . Hence, in any run of size 7 or more, all three must be distinct integers. By Properties 2 and 3 , in any run of size at least 6 in $P_{2}$ the multiple of 5 must be adjacent to the multiple of 6 . In a run of size 8 or more, the unique multiple of 7 must occur in the intersection of the first 7 integers and the last 7; similarly the unique multiple of 6 must occur in the intersection of the first 6 integers and the last 6 . Hence the multiple of 6 and multiple of 7 differ by at most 4 . By Property 7 , there is no run in which the difference is 4 . By Property $5,141^{[8]}$ is the only run of size at least 8 in which the difference is 2 or 3 . Hence, in every other run of size 8 or more, the difference must be 1 .

Property 10 Except for the maximal run $141^{[8]}$, in any run of size 8 or more in $P_{2}$ the multiple of 6 is of the form $6^{a} 2^{6 b}$ or $6^{a} 3^{6 b}$, where $a \geq 1$ and $b \geq 0$ are integers of opposite parity.

Proof Let $R \neq 141^{[8]}$ be a run of size 8 in $P_{2}$, and let $n$ be its multiple of 6. In some order, its multiples of 5 and 7 are $n-1$ and $n+1$, by Property

9. Put $n=2^{c} 3^{d}$, where $c$ and $d$ are positive integers.

Suppose $5 \mid n-1$ and $7 \mid n+1$. Then $2^{c} 3^{d} \equiv 3^{3 c+d} \equiv 1(\bmod 5)$ and $2^{c} 3^{d} \equiv 3^{2 c+d} \equiv-1(\bmod 7)$, so $3 c+d \equiv 0(\bmod 4)$ and $2 c+d \equiv 3(\bmod 6)$. Hence $c \equiv d(\bmod 12)$ and $c \equiv d \equiv 1(\bmod 2)$. Put $a=\min \{c, d\}$, where $a \geq 1$ is odd. Also let $|c-d|=12 s$, and put $\max \{c, d\}=a+6 b$, where $b=2 s \geq 0$ is even. Then $n=6^{a} 2^{6 b}$ or $6^{a} 3^{6 b}$.

Now consider $5 \mid n+1$ and $7 \mid n-1$. In this case $3 c+d \equiv 2(\bmod 4)$ and $2 c+d \equiv 0(\bmod 6)$, so $c \equiv d+6(\bmod 12)$ and $c \equiv d \equiv 0(\bmod 2)$. Put $a=\min \{c, d\}$, where $a \geq 2$ is even. Also let $|c-d|=12 s+6$, and put $\max \{c, d\}=a+6 b$, where $b=2 s+1 \geq 1$ is odd. Again we have $n=6^{a} 2^{6 b}$ or $6^{a} 3^{6 b}$. The property follows.

The combined weight of Properties 5 to 10 now enables us to prove

Theorem 2 In $P_{2}$, up to $10^{25}$ there is no run of size 9 and the only maximal runs of size 8 are $141^{[8]}$ and $212^{[8]}$. 
Proof Property 10 provides a strong restriction on the possible multiples of 6 in any run of size 8 or 9 in $P_{2}$. Up to $10^{25}$ there are just 90 of the special multiples of 6 with $a$ odd, and 84 multiples with $a$ even. It is a straightforward computation to check beside these 174 numbers. We find one gem, the maximal run $212^{[8]}$. No later run of size 8 or 9 occurs up to $10^{25}$.

Indeed, among runs in $P_{2}$ that contain three consecutive integers which are multiples of 5, 6 and 7 , the only other instances of size greater than 3 below $10^{25}$ are $2302^{[7]}$ and $24575^{[5]}$. These computations provide strong evidence for Conjecture 1 .

The study of maximal runs in $P_{2}$ raises other intriguing questions, including: Are there infinitely many pairs of consecutive integers in $P_{2}$ ? What is the smallest positive integer $r_{0}$ such that for each $r \geq r_{0}$ there are only finitely many runs of size $r$ in $P_{2}$ ? We simply don't know the answers to these questions, just as we don't know the answers to the corresponding questions for $P_{1}$.

Next we turn our attention to runs in $P_{n}$ for $n \geq 3$.

\section{Computing runs in $P_{n}$ for $n \geq 3$}

Computation sheds some interesting light on runs in $P_{n}$ with $n \geq 3$, and turns up some delightful gems.

Since $a$ and $a+1$ are coprime, if $\{a, a+1\} \subset P_{n}$, then each has $n$ principal divisors, so $a(a+1) \geq p_{1} p_{2} \cdots p_{2 n}$, the product of the first $2 n$ primes. But $(a+1)^{2}>a(a+1)$, so $a+1>\left(p_{1} p_{2} \cdots p_{2 n}\right)^{1 / 2}$, and the ceiling of this square root is a lower bound for $a+1$. The product of primes is never a square, so the floor of the square root gives the lower bound

$$
a \geq\left\lfloor\left(p_{1} p_{2} \cdots p_{2 n}\right)^{1 / 2}\right\rfloor .
$$

Table 2 lists the first maximal run in $P_{n}$ for $3 \leq n \leq 7$, together with the corresponding factorizations. In each case the first maximal run has size 2. It is interesting to notice how the small primes crowd in and form the majority of principal divisors in the first maximal run of each rank set. It is possible that the first maximal run in $P_{n}$ always occurs in the interval $\left[A, 2^{n} A\right]$, where $A=\left\lfloor\left(p_{1} p_{2} \cdots p_{2 n}\right)^{1 / 2}\right\rfloor$. Indeed, each instance in Table 2 occurs in the interval $\left[A, 2^{n / 2} A\right]$.

Table 3 lists the starters of successive maximal runs of size 2 in $P_{n}$ for $3 \leq n \leq 7$. In each case the interval $\left[A, 2^{n / 2} A\right]$ contains at least 5 


\begin{tabular}{|r|r|r|r|}
\hline$P_{n}$ & First maximal run & $a$ & $a+1$ \\
\hline$P_{3}$ & $230^{[2]}$ & 2.5 .23 & 3.7 .11 \\
$P_{4}$ & $7314^{[2]}$ & 2.3 .23 .53 & 5.7 .11 .19 \\
$P_{5}$ & $254540^{[2]}$ & $2^{2} .5 .11 .13 .89$ & 3.7 .17 .23 .31 \\
$P_{6}$ & $11243154^{[2]}$ & 2.3 .13 .17 .61 .139 & 5.7 .11 .19 .29 .53 \\
$P_{7}$ & $965009045^{[2]}$ & 5.7 .11 .13 .23 .83 .101 & 2.3 .17 .29 .41 .73 .109 \\
\hline
\end{tabular}

Table 2: First maximal runs $a^{[2]}$

maximal runs. Moreover, for $P_{8}$, the corresponding interval contains the maximal run $a^{[2]}$ with $a=68971338435$, since $a=3.5 .17 .23 .29 .31 .103 .127$ and $a+1=2^{2} \cdot 7.11 .13 .19 .37 .107 .229$, but we do not know whether this is the first maximal run in $P_{8}$, nor how many maximal runs occur in this interval.

\begin{tabular}{|l|l|}
\hline$P_{n}$ & Starters of successive maximal runs of size 2 \\
\hline$P_{3}$ & $230,285,429,434,455,494,560,594,609,615, \cdots$ \\
$P_{4}$ & $7314,8294,8645,9009,10659,11570,11780,11934,13299, \cdots$ \\
$P_{5}$ & $254540,310155,378014,421134,432795,483405,486590, \cdots$ \\
$P_{6}$ & $11243154,13516580,16473170,16701684,17348330,19286805, \cdots$ \\
$P_{7}$ & $965009045,1068044054,1168027146,1177173074,1209907985, \cdots$ \\
\hline
\end{tabular}

Table 3: Successive maximal runs of size 2

We have also computed the first maximal runs of various sizes $r \geq 3$ in $P_{n}$ for $3 \leq n \leq 6$. Table 4 summarizes this data.

From Tables 2 and 4 we have $r(3) \geq 16, r(4) \geq 12, r(5) \geq 5, r(6) \geq 3$ and $r(7) \geq 2$. We have also seen that $r(8) \geq 2$. It is noteworthy that the data in Table 4 is not monotonic: the first maximal run of size 14 in $P_{3}$ precedes the first maximal runs of sizes 12 and 13, and the first maximal run of size 7 in $P_{4}$ precedes the first maximal run of size 6 . We have already seen this phenomenon in $P_{2}$, where the first maximal run of size 8 precedes the first maximal run of size 7 .

Let us briefly consider lower bounds for the starters of runs of size $r \geq 3$. Of course, the square root lower bound $A$ for runs of size 2 is also a lower bound for longer runs, but we want something stronger. Suppose $\{a, a+$ $1, a+2\} \subset P_{n}$. The only divisor that can be common to two of these integers is 2 . If $2 \mid a+1$ there is no common divisor, so $a(a+1)(a+2) \geq p_{1} p_{2} \cdots p_{3 n}$, the product of the first $3 n$ primes. If $2 \mid a$ then 2 is a common divisor and $8 \mid a(a+2)$, so $a(a+1)(a+2) \geq 4 p_{1} p_{2} \cdots p_{3 n-1}$. Combining $(a+1)^{2}>a(a+2)$ 


\begin{tabular}{|r|r|r|r|r|}
\hline$r$ & $P_{3}$ & $P_{4}$ & $P_{5}$ & $P_{6}$ \\
\hline 2 & 230 & 7314 & 254540 & 11243154 \\
3 & 644 & 37960 & 1042404 & 323567034 \\
4 & 1308 & 134043 & 21871365 & $\ldots$ \\
5 & 2664 & 357642 & 129963314 & \\
6 & 6850 & 2713332 & 830692265 & \\
7 & 10280 & 1217250 & 4617927894 & \\
8 & 39693 & 14273478 & $\ldots$ & \\
9 & 44360 & 44939642 & & \\
10 & 48919 & 76067298 & & \\
11 & 218972 & 163459742 & & \\
12 & 534078 & 547163235 & & \\
13 & 2699915 & 2081479430 & & \\
14 & 526095 & 2771263512 & & \\
15 & 17233173 & $\ldots$ & & \\
16 & 127890362 & & & \\
\hline
\end{tabular}

Table 4: First maximal runs of increasing size

with the very weak inequality $p_{3 n}>4$ yields a lower bound that holds regardless of whether $a$ is odd or even:

$$
a \geq\left\lfloor 2\left(p_{2} p_{3} \cdots p_{3 n-1)}\right)^{1 / 3}\right\rfloor .
$$

Let us denote this lower bound by $B$. Similar reasoning yields lower bounds for the starters of longer runs, but here we only consider $A$ and $B$.

If $n=2$ then $A=14$ and $B=20$, and these are precisely the starters of the first runs of sizes 2 and 3 in $P_{2}$. If $n=3$ then $230^{[2]} \subset[A, 2 A]$ and $644^{[3]} \subset[B, 2 B]$ where $A=173$ and $B=338$. And so on. Perhaps the first maximal run of size 3 in $P_{n}$ always occurs in the interval $\left[B, 2^{n} B\right]$.

\section{$7 \quad$ Nontrivial runs in $P_{n}$ for $n \geq 3$}

Our computational results certainly confirm that $r(n) \geq 2$ for $n \leq 8$. But it is not obvious that there are nontrivial runs in $P_{n}$ for every $n$. In this section we shall show how to make new runs from old, in particular, how to use suitable sets of 4 neighboring integers in $P_{n}$ to produce pairs of consecutive integers in $P_{2 n-1}$.

For positive integers $a, s, t$ with $s<t$, the integers $a(a+s+t)$ and $(a+s)(a+t)$ differ by $s t$. By imposing appropriate conditions on the divisors 
of $a, a+s, a+t$ and $a+s+t$, we can ensure that

$$
b=\frac{1}{s t} a(a+s+t), \quad b+1=\frac{1}{s t}(a+s)(a+t)
$$

are consecutive integers with equally many principal divisors. The simplest case is when $s=1$ and $t=2$ :

Theorem 3 If $\{a, a+1, a+2, a+3\} \subset P_{n}$ and $12 \mid a(a+3)$, then $b=\frac{1}{2} a(a+3)$ and $b+1=\frac{1}{2}(a+1)(a+2)$ are consecutive integers in $P_{2 n-1}$.

Proof Since $a$ and $a+3$ differ by 3 , their only possible nontrivial common factor is 3 . If $12 \mid a(a+3)$ then exactly one of $a$ and $a+3$ is divisible by 4 , the other is odd and both are divisible by 3 . Hence the principal divisors of $b=\frac{1}{2} a(a+3)$ include a power of 2 , and the product of powers of 3 in $a$ and $a+3$, so $b \in P_{2 n-1}$. Also $a+1$ and $a+2$ are relatively prime and $b+1$ is odd, so $b+1 \in P_{2 n-1}$.

For example, with $a=33$ and $(s, t)=(1,2)$, the run $33^{[4]} \subset P_{2}$ yields $b=594=\frac{1}{2} \cdot 33.36=2.3^{3} .11$ and $b+1=595=\frac{1}{2} \cdot 34.35=5.7 .17$, so $\{b, b+1\} \subset P_{3}$. In fact, $594^{[2]}$ is a maximal run in $P_{3}$.

By making other choices for $a, s, t$ so that $\{a, a+s, a+t, a+s+t\} \subset P_{n}^{*}$, we can construct $\{b, b+1\}=\{a(a+s+t) / s t,(a+s)(a+t) / s t\} \subset P_{m}$, where $P_{m}=P_{2 n-1}$ when $P_{n}^{*}=P_{n}$, and $P_{m}=P_{2 n}$ when $P_{n}^{*}$ is a suitable union of two or more rank sets. Table 5 summarizes some illustrative examples, based on the maximal runs $33^{[4]}, 141^{[8]}$ and $2302^{[7]}$ in $P_{2}$, and $1308^{[4]}$, the first run of size 4 in $P_{3}$. Note that extending into the neighborhood of $2302^{[7]}$ yields some pairs $\{b, b+1\}$ in $P_{4}$.

Up till this point in our discussions, $P_{8}$ is the highest rank set in which we have noted a nontrivial run. With Theorem 3 we can now produce an example in $P_{9}$. The first maximal run of size 4 in $P_{5}$ is $a^{[4]}$ where $a=21871365$. Since $a+3$ is a multiple of 12 , we have

$$
\begin{gathered}
b=\frac{1}{2} a(a+3)=2^{2} .3^{3} .5 .29 .31 .41 .137 .239 .367 \\
b+1=\frac{1}{2}(a+1)(a+2)=7.11 .17 .23 .37 .61 .97 .131 .277 \\
\text { so }\{b, b+1\} \subset P_{9} \text { with } b=239178336288660 . \text { Hence } r(9) \geq 2 .
\end{gathered}
$$

\section{Matched primes in arithmetic sequences}

In this section we shall show how the simultaneous occurrence of primes in two arithmetic sequences leads to pairs of consecutive integers in $P_{n}$. 


\begin{tabular}{|c|cccc|c|}
\hline$P_{n}^{*}$ & $s$ & $t$ & $a$ & $b$ & $P_{m}$ \\
\hline$P_{2}$ & 1 & 2 & 33 & 594 & $P_{3}$ \\
$P_{2}$ & 1 & 2 & 141 & 10152 & $P_{3}$ \\
$P_{2}$ & 1 & 2 & 144 & 10584 & $P_{3}$ \\
$P_{2}$ & 1 & 3 & 141 & 6815 & $P_{3}$ \\
$P_{2}$ & 1 & 3 & 144 & 7104 & $P_{3}$ \\
$P_{2}$ & 1 & 2 & 2304 & 2657664 & $P_{3}$ \\
$P_{2}$ & 1 & 3 & 2303 & 1771007 & $P_{3}$ \\
$P_{2}$ & 1 & 3 & 2304 & 1772554 & $P_{3}$ \\
$P_{2}$ & 1 & 4 & 2303 & 1328831 & $P_{3}$ \\
$P_{2} \cup P_{3}$ & 1 & 3 & 2300 & 1766400 & $P_{4}$ \\
$P_{2} \cup P_{5}$ & 2 & 3 & 2310 & 891275 & $P_{4}$ \\
$P_{2} \cup P_{3}$ & 2 & 3 & 2313 & 893589 & $P_{4}$ \\
$P_{3}$ & 1 & 2 & 1308 & 857394 & $P_{5}$ \\
\hline
\end{tabular}

Table 5: New runs from old

Our approach is based on Dirichlet's famous 1837 theorem on primes in an arithmetic sequence. His presentation can be read in [3]; for a more accessible account $[2]$ is recommended.

Dirichlet's Theorem on Primes in an Arithmetic Sequence For any coprime positive integers $m$ and $r$, the arithmetic sequence $\{k m+r: k \geq 0\}$ contains infinitely many primes.

In fact it is known that the primes are shared rather equitably among the arithmetic sequences with common difference (modulus) $m$. There are $\varphi(m)$ such sequences in which the members are coprime with $m$, and each contains about $n / \varphi(m)$ of the first $n$ primes that are not divisors of $m$. As $n$ grows this is increasingly accurate. Indeed, there is a "folk theorem" that among the primes there are infinitely many occurrences of any finite "pattern" that is not explicitly ruled out by modular considerations. For instance, the patterns $\{a, a+1\}$ and $\left\{a, 2^{a}+1\right\}$ are ruled out modulo 2 and 3 respectively, but $\{a, a+2\}$ and $\left\{a, 2^{a}-1\right\}$ are "possible" patterns in the primes, and the "folk theorem" applied to them would imply the existence of infinitely many twin primes and infinitely many Mersenne primes. Of course, this does not prove these possibilities, but does suggest that they could be true. With such considerations, if we match up the $n$th terms of any two arithmetic sequences that contain primes, it is plausible that infinitely often the matched pairs are both primes. Formally, this can be stated as follows: 
Conjecture 2 If $m, m^{\prime}, r, r^{\prime}$ are positive integers with $\operatorname{gcd}\{m, r\}=1$ and $\operatorname{gcd}\left\{m^{\prime}, r^{\prime}\right\}=1$, there are infinitely many positive integers $k$ such that $k m+r$ and $\mathrm{km}^{\prime}+r^{\prime}$ are both prime.

For example, $2 k+1$ and $3 k+2$ are simultaneously prime when $k=$ $1,3,5,9,15, \cdots$, and the corresponding pairs are $\{2 k+1,3 k+2\}=\{3,5\}$, $\{7,11\},\{11,17\},\{19,29\},\{31,47\}, \cdots$. This is of interest in our present context because $b=3(2 k+1)$ and $b+1=2(3 k+2)$ are consecutive integers, so $\{b, b+1\} \subset P_{2}$ whenever $2 k+1$ and $3 k+2$ are primes greater than 3 . We deduce that $\{21,22\},\{33,34\},\{57,58\},\{93,94\}, \cdots$ are pairs of consecutive integers in $P_{2}$. Generalizing this example, we have

Theorem 4 If Conjecture 2 is true, there are infinitely many pairs of consecutive integers in $P_{n}$, for each $n \geq 2$.

Proof For some $n \geq 2$ let $m=p_{1} \cdots p_{n-1}$ and $m^{\prime}=p_{n} \cdots p_{2 n-2}$ be the product of the first $n-1$ primes and the next $n-1$ primes, respectively. Let $x_{0}$ be the smallest positive solution of the simultaneous congruences $x \equiv 1(\bmod m)$ and $x \equiv 0\left(\bmod m^{\prime}\right)$. The Chinese Remainder Theorem (see [16], for example) ensures that $x_{0}$ exists, and the general solution is $x \equiv x_{0}\left(\bmod m m^{\prime}\right)$. Then there are positive integers $r$ and $r^{\prime}$ such that $x_{0}-1=m r^{\prime}$ and $x_{0}=m^{\prime} r$, so the general solution satisfies

$$
\begin{aligned}
x & =m^{\prime} r+k m m^{\prime}=m^{\prime}(k m+r) \\
x-1 & =m r^{\prime}+k m m^{\prime}=m\left(k m^{\prime}+r^{\prime}\right)
\end{aligned}
$$

where $k$ runs through the integers. Since $\operatorname{gcd}\left\{x_{0}, x_{0}-1\right\}=1$ we have $\operatorname{gcd}\left\{m r^{\prime}, m^{\prime} r\right\}=1, \operatorname{sog} \operatorname{gcd}\{m, r\}=\operatorname{gcd}\left\{m^{\prime}, r^{\prime}\right\}=1$. If Conjecture 2 holds, it follows that there are infinitely many positive integers $k$ such that $p=k m+r$ and $q=k m^{\prime}+r^{\prime}$ are simultaneously prime. If $k$ is large enough, then $p$ and $q$ are not among the first $2 n-2$ primes, so $b=m q$ and $b+1=m^{\prime} p$ are consecutive integers with $n$ principal divisors (all of which happen to be prime).

Although Theorem 4 depends on the unproven Conjecture 2, it is still effective in yielding numerical results, because the proof shows how computation can be used to seek concrete instances of the construction. For example, when $n=10$ we have

$$
\begin{aligned}
m & =2 \cdot 3 \cdot 5 \cdot 7 \cdot 11 \cdot 13 \cdot 17 \cdot 19 \cdot 23=223092870 \\
m^{\prime} & =29 \cdot 31 \cdot 37 \cdot 41 \cdot 43 \cdot 47 \cdot 53 \cdot 59 \cdot 61=525737919635921 .
\end{aligned}
$$


The smallest positive solution to $x \equiv 1(\bmod m)$ and $x \equiv 0\left(\bmod m^{\prime}\right)$ is

$$
x_{0}=6949903578918639188851,
$$

so $x_{0}-1=m r^{\prime}$ and $x_{0}=m^{\prime} r$ give $r=13219331$ and $r^{\prime}=31152513206355$. The sequences $\{k m+r: k \geq 0\}$ and $\left\{k m^{\prime}+r^{\prime}: k \geq 0\right\}$ are simultaneously prime when $k=26,38,74, \cdots$, so the smallest pair of matched primes is $\{p, q\}=\{5813633951,13700338423740301\}$ yielding $\{b, b+1\}=$ $\left\{m q, m^{\prime} p\right\} \subset P_{10}$ with $b=3056447818923499884753870$. Hence $r(10) \geq 2$.

\section{$9 \quad$ Upper bounds on $r(n)$ for $n \geq 3$}

In the Introduction we noted that no run of consecutive integers in $P_{n}$ can contain a multiple of $M=p_{1} p_{2} \cdots p_{n+1}$, the product of the first $n+1$ primes, so $r(n)<M$. Thus $r(2)<30$. Eventually we proved $8 \leq r(2) \leq 9$. As a first step toward this result, we showed that no run in $P_{2}$ contains more than one multiple of 6 , which immediately implies the improved upper bound $r(2)<12$. We shall now show that the same ideas yield corresponding results for $n \geq 3$.

Theorem 5 For any positive integer $n$, let $N=p_{1} p_{2} \cdots p_{n}$ be the product of the first $n$ primes, and let $b$ be the largest integer such that no prime factor of the product $b(b+1)$ exceeds $p_{n}$. Then no run of consecutive integers greater than $b N$ in $P_{n}$ contains more than one multiple of $N$.

Proof For any integer $b$, if no prime factor of the product $b(b+1)$ exceeds $p_{n}$, then $\{b, b+1\} \subset S\left(p_{1}, p_{2}, \cdots, p_{n}\right)$ and conversely. By the Neighboring Pairs Theorem, there exists a largest integer $b$ with this property. Then $b N$ and $(b+1) N$ are the last two consecutive multiples of $N$ in $S\left(p_{1}, p_{2}, \cdots, p_{n}\right)$. Suppose $R$ is a run of consecutive integers greater than $b N$ in $P_{n}$, and suppose $R$ contains at least one multiple of $N$. Let $a N$ be the smallest multiple of $N$ in $R$. Then $a N \in P_{n}$ and $N \in P_{n} \cap S\left(p_{1}, p_{2}, \cdots, p_{n}\right)$, so $a \in S\left(p_{1}, p_{2}, \cdots, p_{n}\right)$. But $a>b$, so $a+1 \notin S\left(p_{1}, p_{2}, \cdots, p_{n}\right)$. Thus $a+1$ contains a prime factor $p>p_{n}$, and $(a+1) N$ has at least $n+1$ principal divisors. Thus $(a+1) N \notin P_{n}$, so $(a+1) N \notin R$, and the theorem follows.

Corollary 3 No run of consecutive integers in $P_{3}$ contains more than one multiple of 30 , and no run of consecutive integers in $P_{4}$ contains more than one multiple of 210. 
Proof Since $N=30$ is the product of the first 3 primes, and $b=80$ is the largest integer such that $\{b, b+1\} \subset S(2,3,5)$, the theorem ensures that no run of consecutive integers greater than $b N=2400$ contains more than one multiple of 30 . On the other hand, the gap between each pair of consecutive primes up to 2411 , the first prime greater than $b N$, is less than 30 , with one exception. The exceptional pair is $\{1327,1361\}$, with difference 34. Since 1350 is the only multiple of 30 between 1327 and 1361, it follows that among the nonnegative integers up to $(b+1) N=2430$, there is at least one prime between every pair of consecutive multiples of 30 . Hence no run of consecutive integers in $P_{3}$ contains two consecutive multiples of 30 .

A similar argument applies for $P_{4}$, with $N=210$ and $b=4374$. The gaps between consecutive primes up to 918563 , the first prime greater than $b N$, are all less than 210 , so the claimed result follows. Indeed, the largest gap between consecutive primes up to 918563 is 114, achieved by the pair $\{492113,492227\}$.

Corollary 3 immediately implies bounds which are better than $r(3)<$ 2.3.5.7 $=210$ and $r(4)<2.3 .5 .7 .11=2310$, but presumably they are still far from the true values.

Corollary 4 Upper bounds on the size of maximal runs in $P_{3}$ and $P_{4}$ are $r(3) \leq 59$ and $r(4) \leq 419$.

\section{Closing remarks}

An extension of Størmer's Neighboring Pairs Theorem shows that for any finite set of primes $P$ and any positive constant $c$ there are only finitely many pairs of integers in $S(P)$ which differ by $c$. As noted in [8], this follows from a theorem of Alan Baker on logarithms of algebraic numbers [1].

One of the most intriguing questions left open in our discussion is whether there are pairs of consecutive integers in $P_{n}$ for every $n \geq 1$. Examples for $1 \leq n \leq 10$ are found in the paper, but what is the case for larger $n$ ? We boldly conjecture that in fact there are infinitely many such pairs for every $n \geq 1$. We showed that this holds for $n \geq 2$ if Conjecture 2 is true. Although Conjecture 2 does not appear to imply that $P_{1}$ contains infinitely many pairs of consecutive integers, it does imply that there are infinitely many twin primes, an assertion that is a notorious unproven conjecture in its own right.

Apart from our computational results, we have shed little light on the existence of runs of size 3 in $P_{n}$. We found such runs for $1 \leq n \leq 6$, but 
we have no example with $n \geq 7$, and no basis for conjecturing whether or not any $P_{n}$ might contain infinitely many such runs. But it is conceivable that for every $n \geq 1$ there are only finitely many runs of size greater than $N$ in $P_{n}$, where $N$ is the product of the first $n$ primes. We showed that this is true when $n=1$, and we gave strong computational evidence in its favor when $n=2$.

Acknowledgements The first author is grateful for hospitality, during much of the work on this paper, from the School of Mathematical and Physical Sciences of The University of Newcastle, where he holds a conjoint professorship. Both authors wish to thank Dr. Jason Kimberley for independent verification of the computational results presented in the various tables.

\section{References}

[1] A. Baker, Linear forms in the logarithms of algebraic numbers (IV), Mathematika 15 (1968), 204-216.

[2] H. Davenport, Multiplicative Number Theory, 2nd ed., 1980, Springer, Berlin.

[3] G. L. Dirichlet, Lectures on Number Theory, trans. J. Stillwell 1999, Amer. Math. Soc., Providence, RI.

[4] E. F. Ecklund, Jr. and R. B. Eggleton, Prime factors of consecutive integers, Amer. Math. Monthly 79 (1972), 1082-1089.

[5] E. F. Ecklund, Jr. and R. B. Eggleton, A note on consecutive composite integers, Math. Mag. 48 (1975), 277-281.

[6] R. B. Eggleton and W. F. Galvin, Upper bounds on the sum of principal divisors of an integer, Math. Mag. 74 (2004), 190-200.

[7] The 44th Mersenne prime, with exponent $n=32582657$, was discovered on Sept. 4, 2006. GIMPS: The Great International Mersenne Prime Search. http://www.mersenne.org/prime.htm

[8] G. D. Halsey and E. Hewitt, More on the superparticular ratios in music, Amer. Math. Monthly 79 (1972), 1096-1100.

[9] D. H. Lehmer, On a problem of Størmer, Illinois J. Math. 8 (1964), 57-79. 
[10] D. H. Lehmer, The prime factors of consecutive integers, Amer. Math. Monthly 72 (1965), no.2, part II, 19-20.

[11] P. Mihailescu, Primary cyclotomic units and a proof of Catalan's conjecture, J. Reine Angew. Math. 572 (2004), 167-195.

[12] T. Nagell, Introduction to Number Theory, 2nd ed. 1964 Chelsea, New York.

[13] C. Størmer, Quelques théorèmes sur l'équation de Pell $x^{2}-D y^{2}= \pm 1$ et leurs applications, Skrifter Videnskabs-selskabet (Christiania) I, Mat.Naturv. Kl., no. 2 (1897), 48pp.

[14] C. Størmer, Sur une équation indéterminée, C. R. Acad. Sci. Paris 127 (1898), 752-754.

[15] C. Størmer, Solution d'un problème curieux qu'on rencontre dans la théorie élémentaire des logarithmes. Nyt Tidsskrift for Mat. B 19 (1908), 1-7.

[16] C. Vanden Eynden, Elementary Number Theory, 2nd ed. 2001 McGrawHill, New York, reissued 2006, Waveland Press, Long Grove, IL. 